\title{
Auf der Jagd nach Ritterspornen in Malawi - Eindrücke von einer Sammel- und Fotoexpedition
}

\author{
Marco Schmidt, Stefan Dressler, Florian Jabbour \& Elke Faust
}

\begin{abstract}
The stations of a botanical expedition from Zomba in the south to the Nyika plateau in the north of Malawi provide insight into the vegetation types and flora of the country.
\end{abstract}

\section{Zusammenfassung}

Die Stationen einer botanischen Sammel- und Fotoexpedition von Zomba im Süden bis zum Nyika-Plateau im Norden Malawis geben einen Einblick in Vegetationstypen und Florenelemente des Landes.

\section{Die Reise}

Unsere Reise hatte zwei Hauptziele. Zwei von uns, Florian Jabbour und Stefan DressLER, hatten Mittel von der National Geographic Society erhalten, um eine Gruppe afromontaner Ritterspornarten und deren Hybriden für ein Forschungsprojekt zu besammeln und zu untersuchen. Außerdem wollten wir die Gelegenheit nutzen, die fotografische Dokumentation der afri-

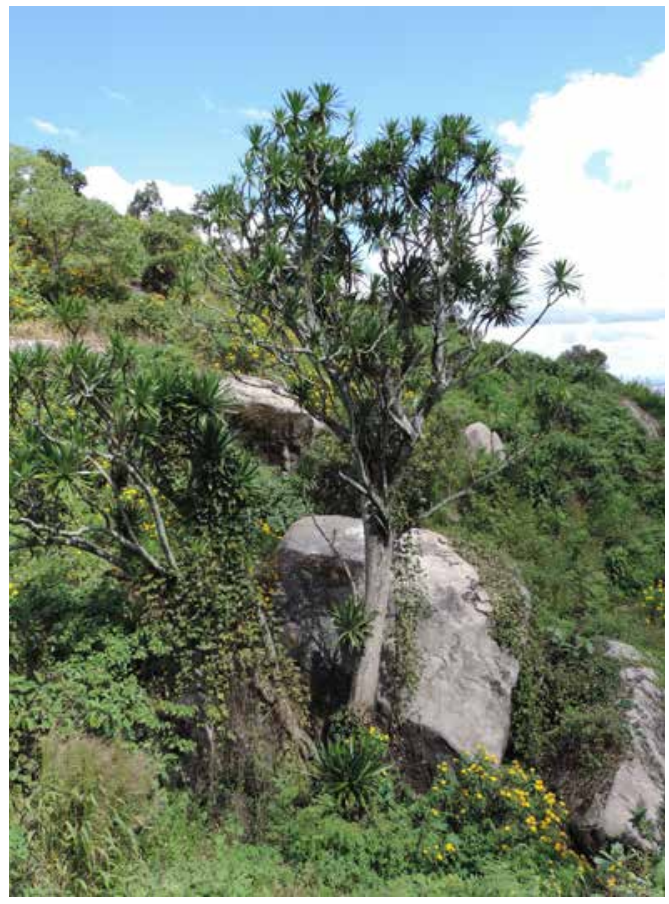

Abb. 1: Dracaena steudneri, Dedza-Berg. Außerdem sind größere Bestände der gelbblühenden Tithonia diversifolia zu sehen. (Foto: M. SснміDT) kanischen Flora für unser Online-Projekt „African Plants - a photo guide“ (DressLer et al. 2014) zu erweitern. Elke Faust, ehrenamtliche Mitarbeiterin in der Botanik bei Senckenberg, hatte jahrelang in Malawi gelebt und bot ihre Unterstützung an. Auch gab es gute Kontakte über Senckenberg und Goethe-Universität zur Universität in Zomba. Und es war bekannt, dass die beiden in Malawi vorkommenden Delphinium leroyi und $D$. dasy-

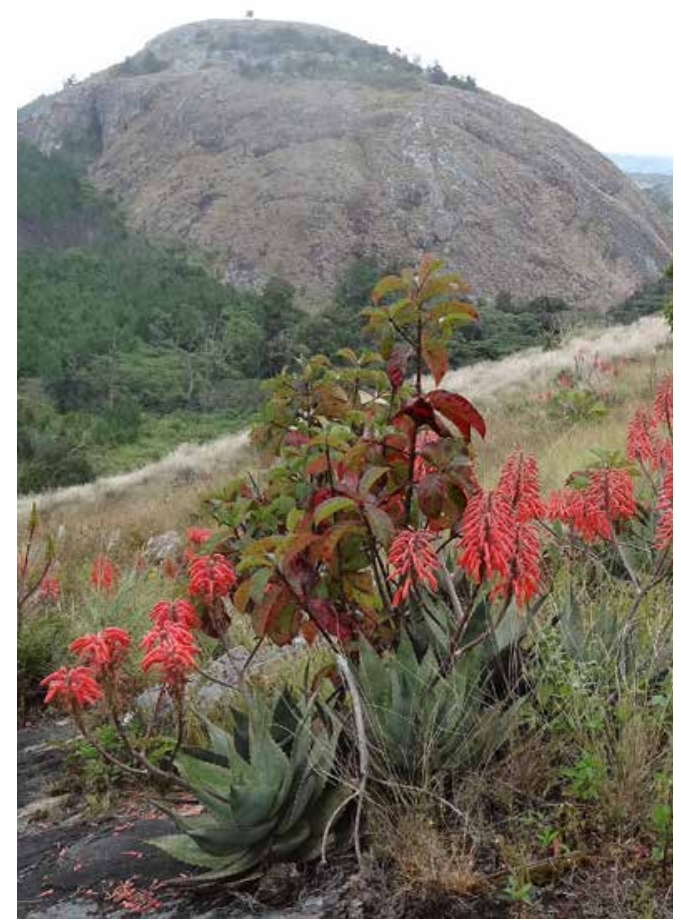

Abb. 2: Aloe chabaudii, Inselberg südlich von Mzuzu.

(Foto: S. Dressler) 


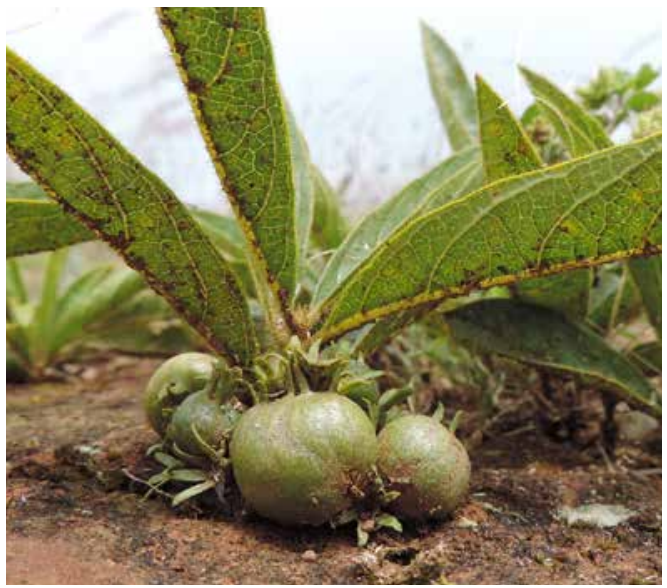

Abb. 3: Gardenia subacaulis, bei Chikangawa.

(Foto: M. SснміDт)

caulon in der Region des Nyika-Plateaus gemeinsam vorkommen und Hybriden bilden (JoHnsON 2001). Im tropischen Afrika kommen nur drei Arten von Ritterspornen vor, die als eher temperate Arten zumeist in größeren Höhenlagen siedeln, quasi auf temperaten Inseln über dem tropischen

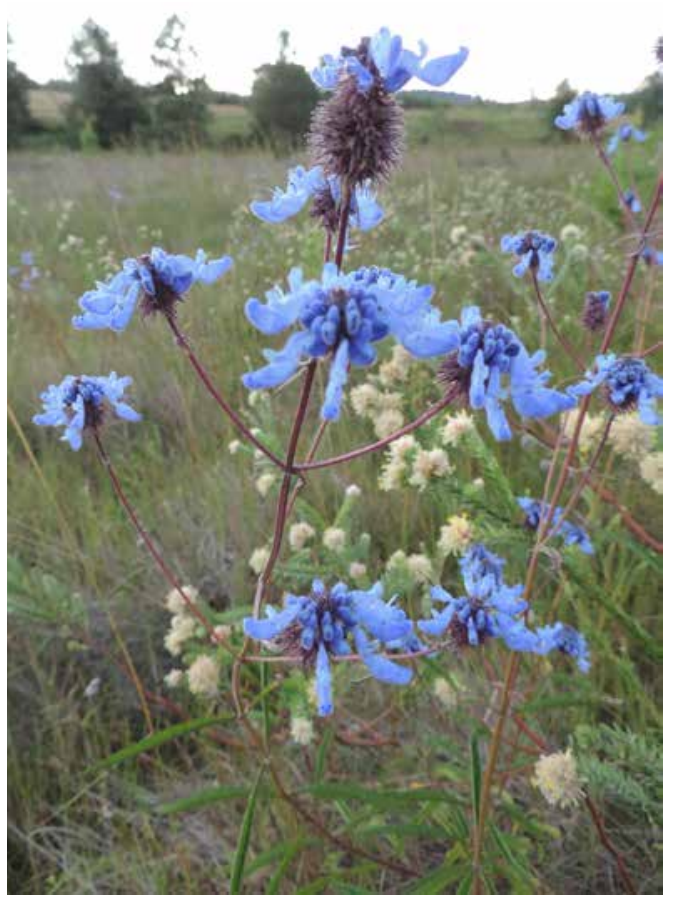

Abb. 4: Pycnostachys stuhlmannii, Dambo nahe der Luwawa Lodge, Vyphia-Berge. Im Bildhintergrung Gnidia mollis. (Foto: M. SснміDт)
Flachland. Diese Arten sind morphologisch gut charakterisiert, genetisch jedoch nicht differenziert (Chartier et al. 2016). Dieses Phänomen ist Gegenstand eines Forschungsprojektes, für das Material gesammelt werden sollte.

Ende April 2019 flogen wir also nach Blantyre im Süden Malawis, trafen unsere Kollegen vom Herbarium in Zomba, holten die notwendigen Forschungs- und Sammelgenehmigungen ein und versorgten uns für die dreiwöchige Reise. Mit Hassam Patel, Mitarbeiter im Nationalherbar und langjährigem Experten der malawischen Flora, war unser Team vollständig. Samt Gepäck passten wir knapp in Elkes kleinen Geländewagen, es konnte losgehen!

\section{Im Botanischen Garten von Zomba}

Noch während der Vorbereitungen zur Expedition konnten wir ein paar kurze botanische Ausflüge unternehmen. Hassam Patel führte uns durch den Botanischen Garten von Zomba, eine wunderschöne Parkanlage mit einigen interessanten Bäumen und einer Gärtnerei, wo unter anderem die für Malawi endemische und vom Aussterben bedrohte Mulanje-Zeder (Widdringtonia whytei) vermehrt wird. Ein Nachmittag auf dem etwa $2000 \mathrm{~m}$ hohen Zomba-Plateau gab uns einen ersten Eindruck der malawischen Flora, mit beeindruckend großen Dracaena steudneri, zahlreichen Farnen (z.B. Ptisana fraxinea, Cyathea sp., Osmunda regalis), und dem an feuchten Felsen am Straßenrand vorkommenden Streptocarpus goetzei. Auffällig war aber auch das Vorkommen vieler als Zier- oder Nutzpflanzen eingeführter Neophyten, wie etwa Rubus ellipticus, dessen Früchte auch auf dem Plateau verkauft wurden, Dahlia imperialis, und der sehr invasiven Tithonia diversifolia, die uns im ganzen Land noch sehr häufig und oft in großen Beständen begegnen sollte.

\section{Richtung Dezda bis zur Hauptstadt}

Von Zomba aus ging es Richtung Dedza. Auf dem Weg, nahe Ntcheu an der Grenze zu Mosambik lag unsere erste Station, von dem ein Vorkommen von Delphinium dasycaulon bekannt war. Die genau entlang der Grenze verlaufende Straße wurde gerade erneuert, der Zugang zum Mvai-Berg war 


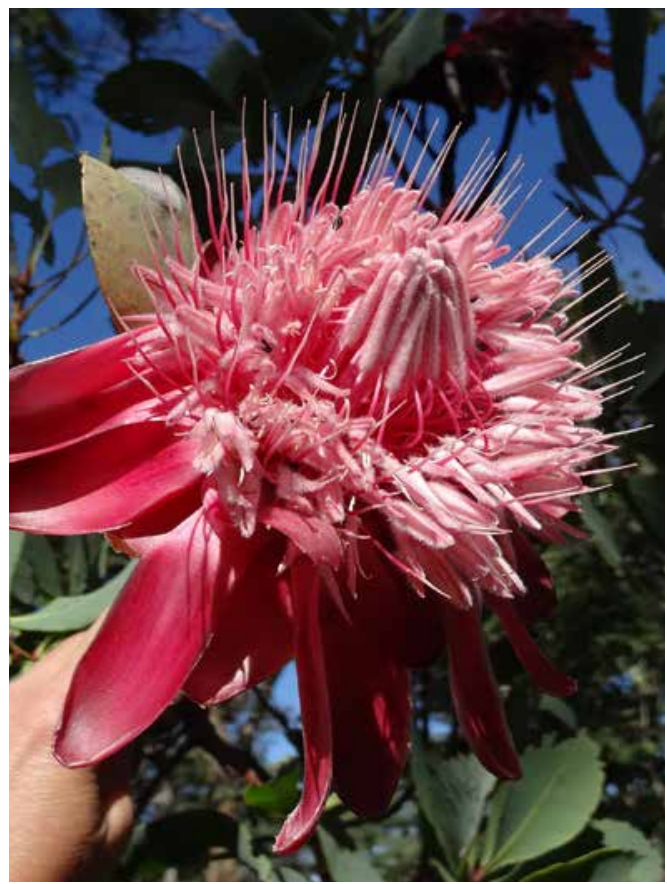

Abb. 5: Protea angolensis var. trichanthera, tiefere Lagen des Nyika-Nationalparks nahe am Thazima Gate.

(Foto: S. Dressler)

mit dem Wagen zunächst nicht möglich, zu Fuß zu zeitaufwändig. Schon auf dem Rückweg zur Hauptstraße haben wir dann doch noch einen Hügel näher erkunden können und tatsächlich unseren ersten Rittersporn gefunden! Am nächsten Tag sind wir dann vom Ort auf den Mt. Dedza gewandert und haben zahlreiche weitere Individuen von $D$. dasycaulon sammeln können. Andere botanische Besonderheiten waren beispielsweise Lippenblütler mit blassvioletten Hochblättern (Haumaniastrum villosum), ein strauchiger Doldenblütler (Heteromorpha arborescens), Diospyros whyteana mit blasigen Früchten, verschiedene Plectranthus-Arten und eine Reihe Vertreter von Gattungen, die wir auch aus unseren Breiten kennen und die im tropischen Afrika vor allem in den kühleren Bergen vorkommen, wie etwa Geranium, Rumex und Rubus.

Als nächstes lag ein Zwischenstopp in der malawischen Hauptstadt Lilongwe an, zwecks Beantragung der Ausfuhrgenehmigung für unsere gesammelten Pflanzen. Bei der Gelegenheit haben

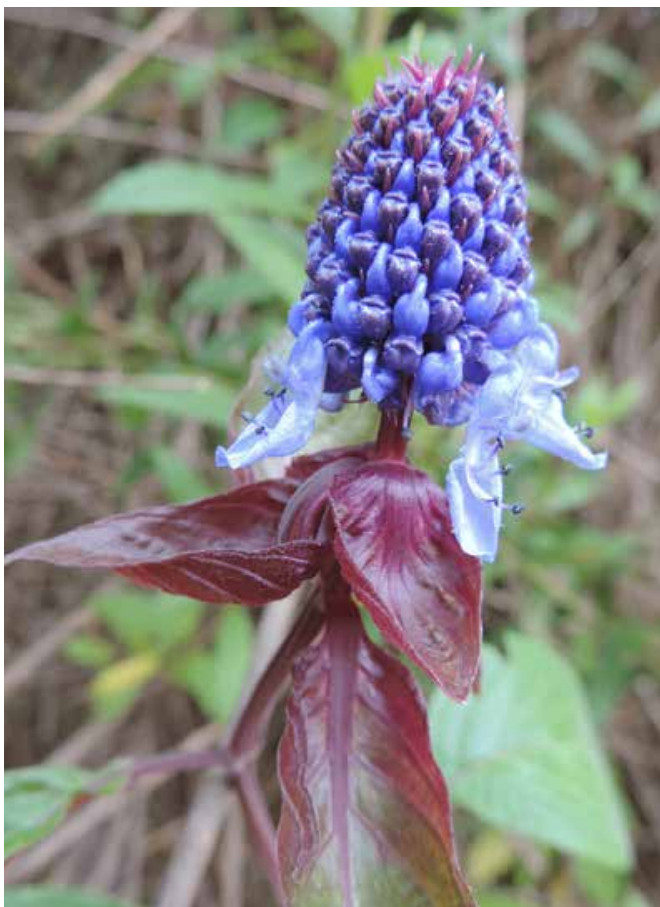

Abb. 6: Pycnostachys urticifolia, tiefere Lagen des Nyika-Nationalparks nahe am Thazima Gate. (Foto: M. SснміDт)

wir auch den Botanischen Garten der Stadt besichtigt, mit einem gut erhaltenen Flecken Baumsavanne in direkter Nachbarschaft der Ministerien und des Parlaments. Außerdem konnten wir hier noch weitere Herbarpressen fürs Pflanzensammeln ausleihen.

\section{Aufbruch zum Nyika-Plateau}

Die Wettervorhersage zwang uns zur Eile, um noch vor Sturm und Starkregen zum Nyika-Plateau $(2000 \mathrm{~m})$ zu kommen, dem wichtigsten Ziel der Sammelreise. Unsere nächste Station sollte in den Vyphia-Bergen sein. Auf dem Weg dorthin machten wir nahe Jenda einen Stopp im Kahingina Forest Reserve mit niedrigen Savannenbäumen wie Strychnos madagascariensis, Ziziphus mucronata und Brachystegia stipulata. Nachmittags kamen wir dann durch dicht mit Flechten bewachsene Brachystegia-Bestände zur Luwawa Forest Lodge. Die niedrigen, lichten Wälder aus nur wenigen Arten, meist Leguminosen, sogenannte Miombo Woodlands, zeigen einen vielfältigen Unterwuchs und sind botanisch sehr interessant. Diese cha- 


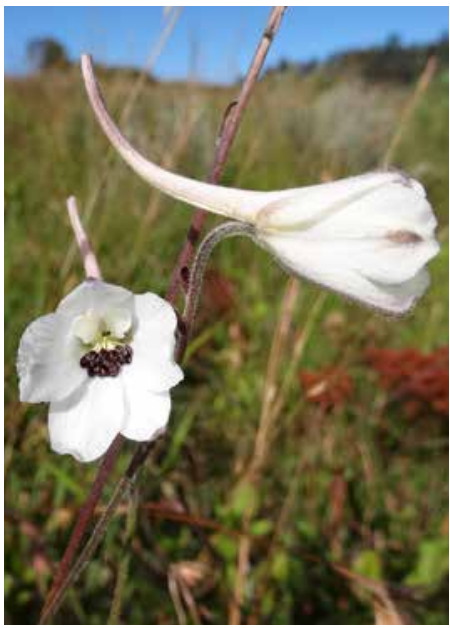

Abb. 7: Delphinium leroyi, Nyika-Plateau (Foto: S. Dressler)

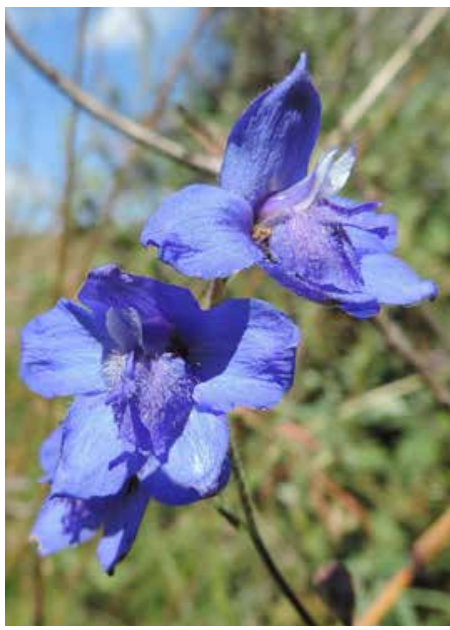

Abb. 8: Delphinium dasycaulon, NyikaPlateau. (Foto: M. SснміDт)

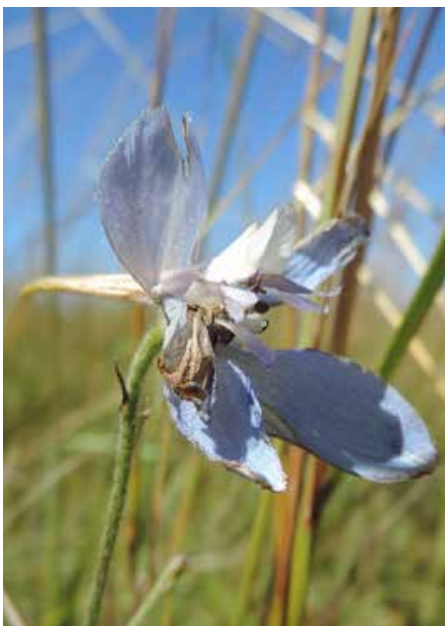

Abb. 9: Delphinum dasycaulon $\times$ leroyi, Nyika-Plateau. Die Hybride liegt in Form und Farbe zwischen den Elternarten. (Foto: M. Sснміdт) rakteristischen Waldsavannen sind im südlichen Zentralafrika weit verbreitet. Ihr Name Miombo nimmt Bezug auf den Lokalnamen der bestandsbestimmenden Brachystegia-Arten. Nahe der Lodge gab es auch die hier Dambo genannten Feuchtwiesen mit sehr schönen Pycnostachys stublmannii, Aeollanthus engleri, Gnidia mollis und einigen Carnivoren (Utricularia reflexa, Genlisea hispidula, Drosera burkeana).

Weiter ging es Richtung Norden. Bei Chikangawa sahen wir uns eine gehölzarme Grasfläche näher an, mit den halbparasitischen Sopubia lanata var. densiflora, zahlreichen Crassula alba, einigen Habenaria kyimbilae und den geoxylischen Gardenia subacaulis. Geoxyle, niedrige Pflanzen mit stark verholzten unterirdischen Organen, treten vermehrt in Savannen des südlichen Afrikas auf und sind meist nah verwandt mit Bäumen und Sträuchern „normaler" Wuchsform (MAURIN et al. 2014). Kurz vor Mzuzu erschienen dann ein paar Inselberge nahe der Straße. Da diese mit ihren speziellen Umweltbedingungen stets interessante Habitate darstellen, haben wir uns dieses Gebiet näher angesehen und wurden nicht enttäuscht: Populationen von Aloe chabaudii in Blüte, die Auferstehungspflanze Myrothamnus flabellifolius, die für Inselberge typische Rubiacee Hymenodic- tyon floribundum und eine Reihe Pflanzen, die in feuchten, dem Fels aufliegenden Humusmatten wachsen, wie Utricularia arenaria, Aeollanthus subacaulis und Lobelia trullifolia.

In Mzuzu bekamen wir mit Bernhard SCHWARz und seinem deutlich robusteren Geländewagen Verstärkung für die kommenden Tage, deckten uns noch mit Verpflegung ein und fuhren nun endlich aufs Nyika-Plateau. Das Plateau liegt zum größten Teil in Malawi, zu einem kleineren in Zambia, ist grenzübergreifend als Nationalpark ausgewiesen und besteht vorwiegend aus montanem Grasland, aber auch einigen beeindruckenden Wäldern. Wir bekamen eine Menge verschiedener Großsäuger zu sehen, so etwa Riedböcke (Redunca arundinum), Pferdeantilopen (Hippotragus equinus), Elenantilopen (Taurotragus oryx), und Crawshay-Zebras (Equus quagga crawshayi). Das Gebiet ist für seine reiche Pflanzenwelt bekannt und umfassend dokumentiert in Plants of the Nyika Plateau (Burrows \& Willis 2005), zu dem auch unser Begleiter Hassam Patel reichlich beigetragen hat. Hier sollte nun also die zweite Ritterspornart sowie Hybriden beider Arten zu finden sein! Und tatsächlich fanden wir schon bald die weißblühende langspornige Delphinium leroyi, meist an flachen Gebüschinseln im Grasland. In 
tieferen Lagen gab es die uns bereits bekannten blauen kurzspornigen Delphinium dasycaulon, und nach langem Suchen und etwas Glück fanden wir auch Pflanzen, die in Form und Farbe zwischen den beiden Arten standen, die also wahrscheinlich Hybriden darstellen!

Für das Plateau können nur einige wenige Arten beispielhaft genannt werden. Im Grasland fanden wir unter anderem Swertia welwitschii, Impatiens assurgens, Protea heckmanniana, Plectranthus acaulis, Kniphofia grantii, Artemisia afra, auf Feuchtwiesen Lobelia mildbraedii, Crepidorhopalon goet$z e i$, in felsigem Gelände Aeollanthus buchnerianus, Tecomaria nyassae, Streptocarpus wittei und Aloe chabaudii. In kleinen Gebüschen kamen Kniphofia princeae, Achyrospermum cryptanthum, Thalictrum rhynchocarpum vor, in den etwas tiefer gelegenen afromontanen Waldinseln zahlreiche Epiphyten an den Baumstämmen, wie etwa Drynaria volkensii, Asplenium mannii, Streptocarpus solenanthus, im Unterwuchs Dracaena laxissima und Cyathea sp. In den tieferen Lagen nahe der Zufahrt standen große schirmförmige Acacia abyssinica und tiefrosa blühende Protea angolensis var. trichantha.

\section{Abstecher zum Malawisee}

Mit den am Nyika-Plateau gesammelten Ritterspornen war das Hauptziel der Sammelexpedition erreicht. Nun konnten wir uns verstärkt der fotografischen Dokumentation der Flora widmen. In Mzuzu verabschiedeten wir uns von Hassam PaTEL und Bernhard SChwarz und fuhren dann geradewegs an den Malawisee, den wir bislang nicht ein einziges Mal gesehen hatten. Nach einem kurzen Stop im eher touristisch geprägten Nkhata Bay ging es weiter am See entlang bis Nkhotakota. Im nahe gelegenen Nkhotakota Wildlife Reserve erkundeten wir unter Begleitung eines Rangers die Savannenvegetation und die Ufer des Bua River. Hyphaene petersiana und Kigelia africana, der Leberwurstbaum, standen gerade in Frucht. Pflanzen mit großen Früchten werden oft von großen Tieren verbreitet und diesen Ausbreitern, nämlich einer Herde Elefanten (Loxodonta africana) sind wir auch bald begegnet: gut, dass wir einen erfahrenen Wildhüter dabeihatten! Sehr beeindruckend waren auch große Bestände des Geweihfarns

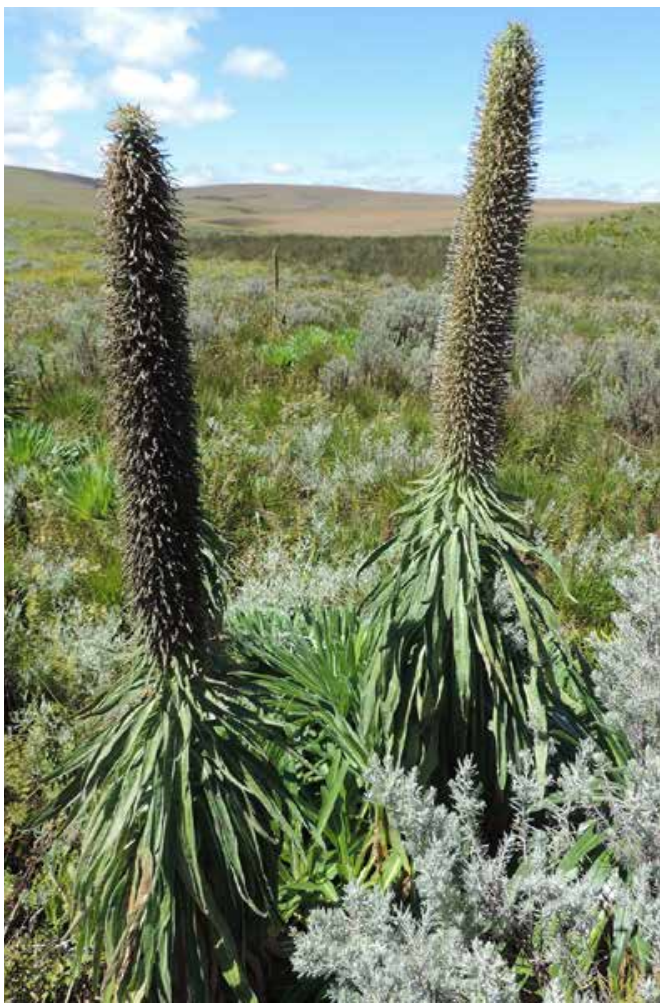

Abb. 10: Lobelia mildbraedii in einem Dambo nahe Chelinda, Nyika-Plateau. (Foto: M. SснміDт)

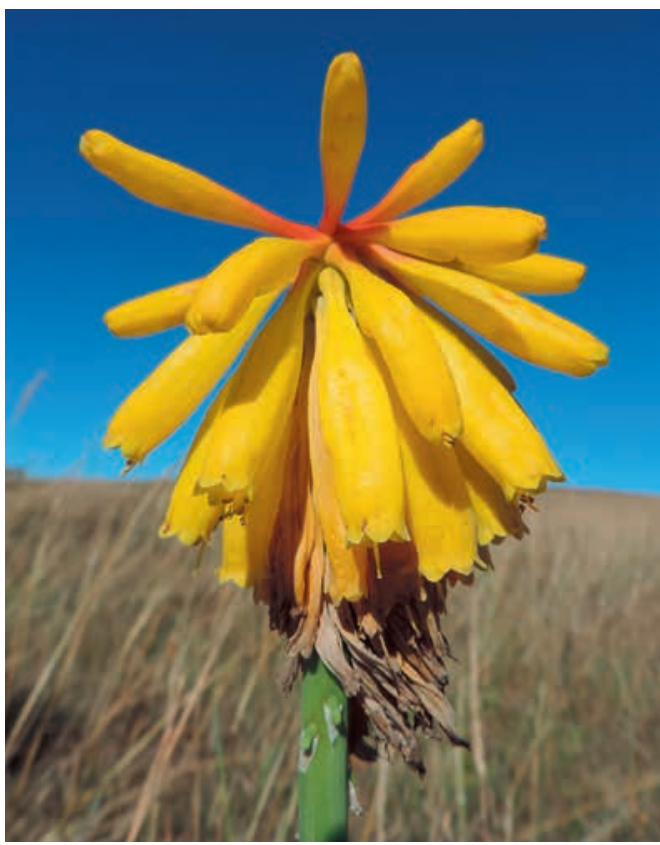

Abb. 11:Kniphofiagrantii, Nyika-Plateau.(Foto:M.SchмIDT) 


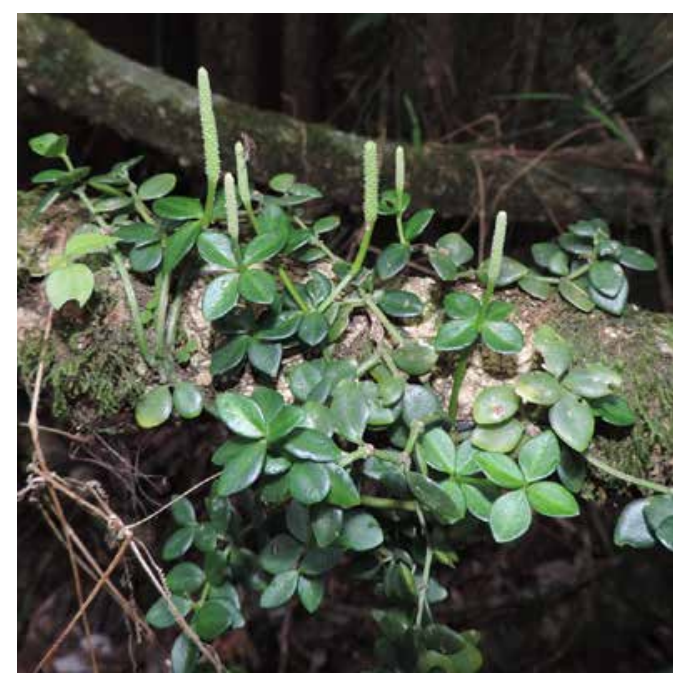

Abb. 12: Peperomia tetraphylla, epiphytisch im Zovochipolo Forest, Nyika-Plateau. (Foto: M. Sснміdт)

Platycerium elephantotis auf Bäumen am Fluss. Später, von unserer Unterkunft aus konnten wir große Schwärme von Eintagsfliegen beobachten, die nach ihrem Schlupf wie dunkle Wolken vom Malawisee aufstiegen.

Unser nächstes Ziel war die Mua Mission. Hier hatte Pater Claude Boucher Chisale ein Kulturzentrum und Museum aufgebaut (www. kungoni.org), wo wir auch übernachten konnten. Der Pater hat sich seit Jahrzehnten um die Bewahrung und Dokumentation der Chewa und anderer Völker in Malawi verdient gemacht und in den letzten Jahren auch vermehrt die Tier- und Pflanzenwelt der näheren Umgebung fotografisch festgehalten. Wir haben auch etwas die Flora der Umgebung erkundet, fanden unter anderem Aloe christianii, Feretia aeruginescens, Sterculia quinqueloba, Pterocarpus angolensis, Stereospermum kunthianum und Sclerocarya birrea (Marula). Ein viel umfassenderer Beitrag zur Dokumentation der afrikanischen Flora sind aber die Fotos von Pater Boucher, die Elke Faust seitdem in den Photo Guide einbindet.

\section{Ntchisi Forest}

Schließlich konnten wir noch ein letztes Ziel anfahren: nach kurzem Halt in Lilongwe ging es in den Ntchisi Forest, ein kleines Regenwaldrelikt

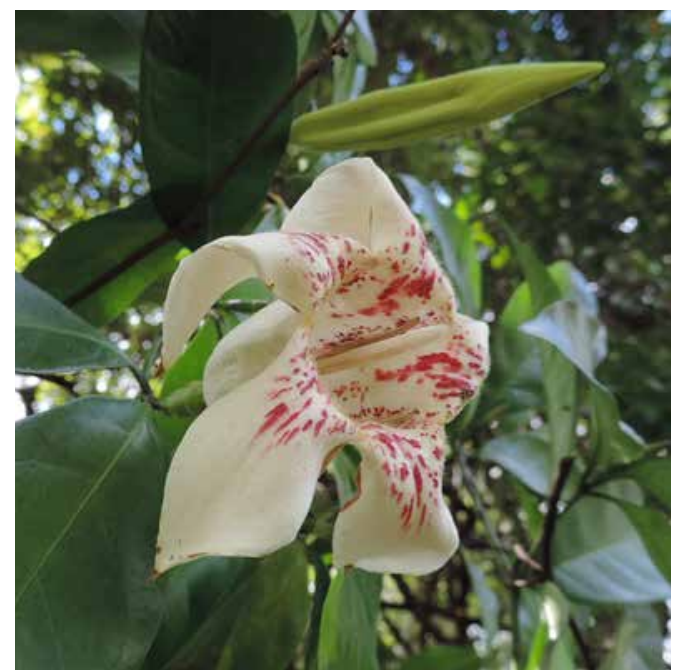

Abb. 13: Rothmannia manganjae, Livulezi Forest. (Foto: M. SснміDт)

auf einem Berg inmitten einer sonst eher kargen Agrarlandschaft und abgepuffert durch umgebende Miombo woodlands. Dieser Regenwald hat sich in der abgelegenen und nicht so dicht besiedelten Gegend erhalten können und trägt auch zur Wasserversorgung der umliegenden Dörfer bei. Leider erlebten wir auch gleich mit, wie die hier eigentlich geschützten Bäume in langen Scheiten als Feuerholz in die umliegenden Dörfer transportiert wurden. Der Wald war reich an Farnen, vor allem zahlreichen Asplenium-Arten. Wir fanden entlang eines Bachlaufs eine dichte

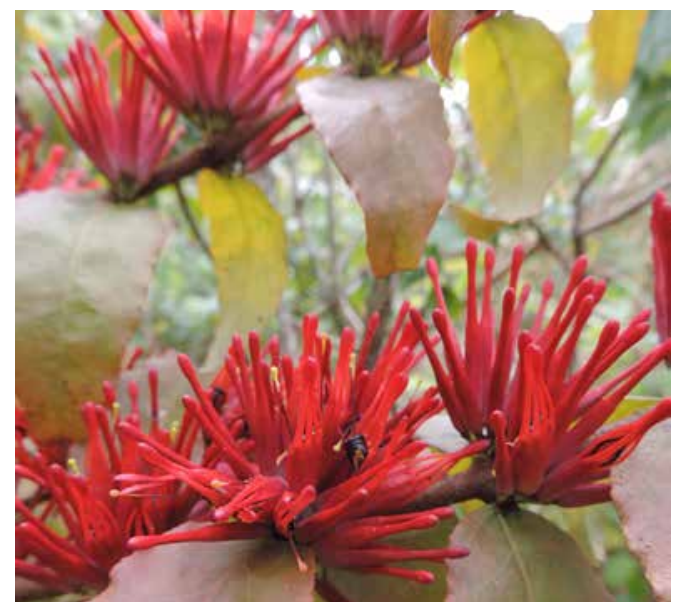

Abb. 14: Englerina inaequilatera, Ntchisi Forest. (Foto: M. SснміDт) 
Population der Abessinischen Banane, Ensete ventricosum, den hier nach der Form seiner Früchte Handgranatenbaum genannten Myrianthus holstii und drei verschiedene Dracaena-Arten, außerdem Piper capense, und Achyrospermum carvalhoi. In den Bäumen sahen wir auch Samango-Affen der seltenen Unterart Cercopithecus albogularis subsp. francescae.

Kurz vor der Präsidentenwahl in Malawi sind wir dann wieder heimgeflogen, eine Menge guter Fotos im Gepäck, ein paar Mitbringsel wie Honig vom Nyika-Plateau und Kaffee aus Mzuzu. Unsere Fotos werden nach und nach in die Bürgerwissenschaftsplattform iNaturalist und unseren Photo Guide integriert. Insgesamt haben wir mehr als 400 verschiedene Arten dokumentieren können, vor allem aber erfolgreich die Rittersporne für unser Forschungsprojekt gefunden, viele freundliche Menschen kennengelernt und wunderbare Eindrücke von Land und Leuten sammeln können.

\section{Literatur}

Burrows, J. E. \& Willis, C. K. (Hrsg.) 2005: Plants of the Nyika plateau: an account of the vegetation of the Nyika National Parks of Malawi and Zambia. Southern African Botanical Diversity Network Report No. 31. - Pretoria.

Chartier, M., Dressler, S., Schönenberger, J., Rojas Mora, A., Sarthou, C., Wang, W. \& Jabbour, F. 2016: The evolution of afro-montane Delphinium (Ranunculaceae): morphospecies, phylogenetics and biogeography. - Taxon 65:1313-1327.

Dressler, S., Schmidt, M. \& ZizKA, G. 2014: Introducing african plants - a photo guide - an interactive identification tool for continental Africa. - Taxon 63: 1159-1161.

Johnson, S. D. 2001: Hawkmoth pollination and hybridization in Delphinium leroyi (Ranunculaceae) on the Nyika plateau, Malawi. - Nordic J. Bot. 21: 599-605.

Maurin, O., Davies, T. J., Burrows, J. E., Daru, B. H., Yessoufou, K., Muasya, A. M., van der Bank, M. \& Bond, W. J.: 2014. Savanna fire and the origins of the 'underground forests' of Africa. - New Phytol. 204: 201-214.

\section{Internetseiten}

www.africanplants.senckenberg.org

www.inaturalist.org/projects/biodiversity-of-malawi

www.kungoni.org

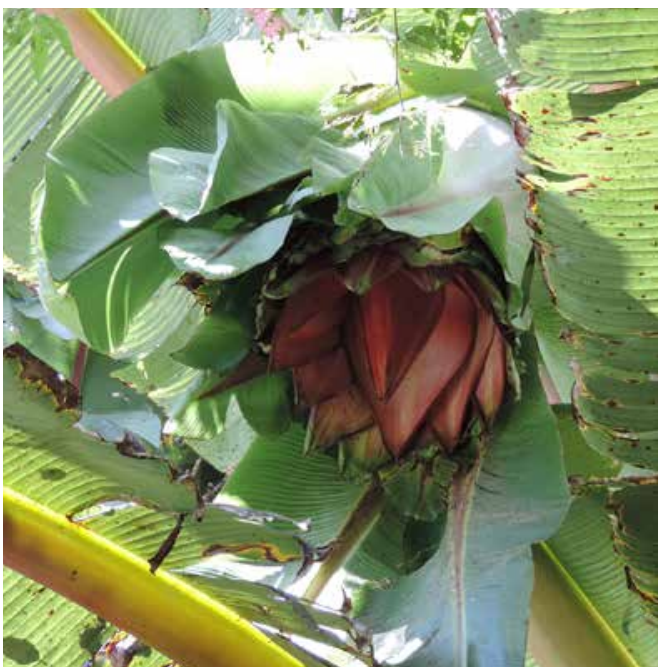

Abb. 15: Blütenstand von Ensete ventricosum, Ntchisi Forest. (Foto: M. ScHMidT)

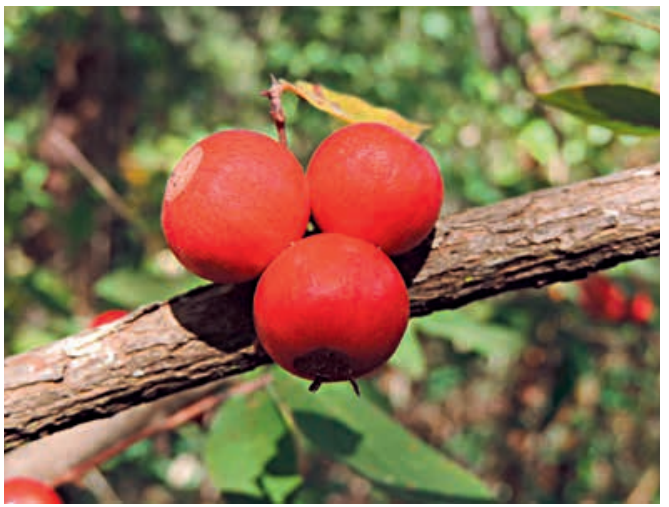

Abb. 16: Feretia aeruginescens nahe der Mua Mission. (Foto: M. SсHмIDT)

\section{Anschriften der Autorin und der Autoren}

Dr. Marco Schmidt, Palmengarten Frankfurt, Siesmayerstr. 61, 60323 Frankfurt,

E-Mail: marco.schmidt@stadt-frankfurt.de

Dr. Stefan Dressler, Senckenberg Forschungsinstitut und Naturmuseum, Senckenberganlage 25, 60325 Frankfurt, E-Mail: stefan.dressler@senckenberg.de

Dr. Florian Jabbour, Musée National d'Histoire Naturelle, 57 rue Cuvier CP39, 75005 Paris, Frankreich, E-Mail: fjabbour@mnhn.fr

Elke Faust, Im Höhlchen 32, 65795 Hattersheim E-Mail: elkemariafaust@web.de 